\title{
Muscle Forces or Gravity: What Predominates Mechanical Loading on Bone?
}

\author{
Wendy M. Kohrt ${ }^{1}$, Daniel W. Barry ${ }^{2}$, and Robert S. Schwartz ${ }^{1}$ \\ ${ }^{1}$ Division of Geriatric Medicine, Department of Medicine, University of Colorado Denver, Aurora, \\ CO \\ 2Division of General Internal Medicine, Department of Medicine, University of Colorado Denver, \\ Aurora, $\mathrm{CO}$
}

\begin{abstract}
Most mechanical forces acting on the skeleton are generated either through impact with the ground (i.e., gravitational loading) or through muscle contractions (i.e., muscle loading). If one of these conduits for activating mechanotransduction in bone is more effective than the other with respect to developing or maintaining bone strength, this would have important clinical implications for prescribing physical activity for the prevention or treatment of osteoporosis. This section of the symposium considered whether there is evidence from studies of humans that the effectiveness of physical activity to preserve bone health is dependent on whether the activities stimulate the skeleton primarily through gravitational or muscle loading. Conclusive evidence is lacking, but several lines of research suggest that physical activities that involve impact forces, and therefore generate both gravitation and muscle loading, are most likely to have beneficial effects on bone metabolism and reduce fracture risk.
\end{abstract}

\section{Keywords}

Bone Mineral Density; Bone Mineral Content; Hip Fracture; Ground-Reaction Force; JointReaction Force; Impact Force

Most of the mechanical forces that act on the skeleton during physical activities are generated either through impact with the ground (i.e., gravitational or ground-reaction forces) or through skeletal muscle contractions (i.e., muscle or joint-reaction forces). If one of these conduits for activating mechanotransduction in bone is more effective than the other with respect to developing or maintaining bone strength, this would have important clinical implications for prescribing physical activity for the prevention or treatment of osteoporosis. The intent of this component of the symposium was to discuss whether there is evidence from clinical research (i.e., studies of humans) that the effectiveness of physical activity to preserve bone health is dependent on whether the activities stimulate the skeleton primarily through gravitational or muscle forces. However, as discussed in other sections of the symposium proceedings, most, if not all, activities that generate gravitational forces also involve muscle forces (e.g., running, jumping). In contrast, some activities stimulate the skeleton almost exclusively through muscle loading because they involve little or no impact with the ground (e.g., weight lifting, swimming). The discussion will focus on the types of

Copyright ( 92009 by the American College of Sports Medicine

Address for correspondence: Wendy M. Kohrt, Ph.D., Mail Stop B179, 12631 E 17th Ave, Room 8111, Aurora, CO 80045; wendy.kohrt@ucdenver.edu . 
activities that have been found in clinical studies to have beneficial effects on bone mineral density (BMD) or to reduce risk for osteoporotic fracture, with emphasis on whether efficacy is related to the loading characteristics of the activity.

\section{TERMINOLOGY}

The terms "non-weight-bearing" and "weight-bearing" are often used in clinical studies to categorize physical activities with respect to their bone-loading potential. However, these terms do not provide a clear distinction as to whether the activity stimulates the skeleton via gravitational and/or muscle forces. As an example, both running and weight lifting are weight-bearing activities, but they differ in the level of impact forces they generate. For the purpose of addressing the topic of this symposium, gravitational loading will be considered to be forces generated as a result of impact with the ground (e.g., running) not as a result of lifting a weight against gravity (e.g., weight lifting). The term "impact" will be used to describe activities that take advantage of the body mass impacting the ground to generate gravitational loading, and the term "no-impact" will be used to describe activities that involve little or no impact with the ground.

\section{ASSOCIATION OF BONE MASS AND MUSCLE MASS}

If the muscle forces generated during either no-impact or impact activities are the primary regulators of bone mass, it would be reasonable to expect that bone mass and muscle mass would be strongly related. This was evaluated by the authors, using fat-free bone-free mass (FFBFM) as a surrogate measure of muscle mass, in an existing data set of 685 women and men, aged 20 to 91 yr (i.e., the Denver cohort), who had volunteered for a variety of research studies in the authors' laboratory and had body composition assessed by dualenergy x-ray absorptiometry. Indeed, total body bone mineral content (TBBMC) was more strongly associated with total body FFBFM (Fig. 1, top panel: women, $r=0.68$; men, $r=$ 0.63 ) than with fat mass (Fig. 1, bottom panel: women, $r=0.22$; men, $r=0.14$ ) or total mass (data not shown; women, $r=0.48$; men, $r=0.45$ ). Even more striking, the linear regression lines describing the associations between TBBMC and FFBFM were almost identical for women and men (Fig. 1, top panel). These findings were very similar to those reported previously by one of the authors in a separate cohort of 562 women and men, aged 55 to 95 yr (i.e., the St. Louis cohort) (4). The strong association of TBBMC with FFBFM, and the similarity of the association in women and men, seemingly provides compelling support for the notion that muscle forces are the primary mediators of the effects of mechanical loading on the skeleton. However, because muscle forces are generated during both no-impact (i.e., muscle forces only) and impact (i.e., gravitational and muscle forces) activities, it is not possible to isolate the potential role of gravitational loading as a mediator of either TBBMC or fat-free mass.

\section{MUSCULOSKELETAL EFFECTS OF NO-IMPACT VERSUS IMPACT EXERCISE}

Evidence that both gravitational and muscle forces are important for skeletal regulation comes from intervention studies that have compared the effects of no-impact versus impact exercise training on BMD $(17,35)$. As discussed by Drs. Judex and Carlson in one of the accompanying papers of the symposium proceedings, premenopausal women who engaged in 8 months of exercise training involving either running or weight lifting had similar increases in lumbar spine BMD (35). However, only the weight lifting group had increases in muscle strength. In a similar study, postmenopausal women who underwent 9 months of either no-impact (i.e., weight lifting, rowing) or impact (i.e., walking, jogging, stair climbing/descending) exercise training had comparable increases in lumbar spine and total 
hip BMD, but only the impact group had increases in femoral neck BMD, which is a common site of osteoporotic fracture (Fig. 2) (17). Women in the no-impact program had an increase in fat-free mass, but women in the impact program did not (Fig. 2).

Both of these intervention studies were consistent with the fundamental concept that increases in muscle mass and strength occur in response to resistance, but not endurance, exercise training (7). However, despite the discordant effects on muscle, both types of exercise programs had similar benefits on BMD in some skeletal regions. This suggests that bone responds either to muscle forces that are below the threshold necessary to stimulate muscle hypertrophy or to gravitational forces generated by impact exercise. Support for the latter comes from the observation that femoral neck BMD increased in response to impact, but not in response to noimpact, exercise (Fig. 2) (17). This finding is consistent with the results of meta-analytic studies of the effects of exercise training on BMD. One metaanalysis that focused only on exercise interventions that included any type of impact activities, either alone or in combination with no-impact activities, found beneficial effects on femoral neck BMD (23). In contrast, meta-analyses conducted by the same investigators that focused only on no-impact exercise training interventions (i.e., resistance exercise) found no significant benefit on femoral neck BMD $(24,25)$. Because of its cantilever-like position to bear the weight of the upper body, the femoral neck region of the proximal femur would be expected to be particularly susceptible to impact loading. In patients with instrumented hip prostheses, as the speed of ambulation increases there are progressive increases in both ground-reaction forces (i.e., magnitude of impact) and in the loading forces on the head and neck region of the prosthesis (3). Thus, activities that generate groundimpact forces would be expected to have beneficial effects on weight-bearing regions of the skeleton as a result of the gravitational forces, per se, and/or the accompanying muscle forces.

\section{MUSCULOSKELETAL EFFECTS OF REDUCED GRAVITATIONAL LOADING}

When gravitational forces are markedly reduced, such as during bed rest or spaceflight, there is a rapid and profound deleterious effect on the skeleton (22). In a classic study of bed rest to simulate the effects of microgravity on BMD, young men had decreases in femoral neck and lumbar spine BMD of 3\%-4\% after only $17 \mathrm{wk}$ (21). Such rates of decline are $~ 10$-fold higher than the accelerated rate of bone loss that occurs at the time of the menopause in women. Because activities that generate gravitational forces also generate muscle forces, the effects of spaceflight and bed rest reflect reductions in both types of loading. As would be expected with marked reductions in muscle forces, considerable muscle atrophy also occurs under these conditions (22). Exercise countermeasures have been partially effective in preventing bone and muscle loss during 2 to 4 months of bed rest $(33,34,36)$. For example, performing a variety of upper-body $\left(3 \mathrm{~d} \cdot \mathrm{wk}^{-1}\right)$ and lower-body $\left(3 \mathrm{~d} \cdot \mathrm{wk}^{-1}\right)$ resistance exercises using a horizontal exercise machine was effective in preserving muscle mass and BMD in some, but not all, body regions (33). Similarly, lower-extremity resistance exercise $\left(2\right.$ or $3 \mathrm{~d} \cdot \mathrm{wk}^{-1}$ ) and supine endurance exercise on a vertically oriented treadmill in a lowerbody negative pressure chamber $\left(2-4 \mathrm{~d} \cdot \mathrm{wk}^{-1}\right)$ attenuated but did not prevent a decline in hip BMD, although quadriceps muscle mass was preserved and strength was increased $(34,36)$. Exercise countermeasures on the International Space Station have also been ineffective at preventing either cortical or trabecular bone loss (20). The findings from bed rest and spaceflight studies indicate that it is very challenging to preserve BMD in weight-bearing regions of the skeleton with no-impact exercises and highlight the important role that gravitational forces play in the maintenance of skeletal mass and integrity. 


\section{WEIGHT-SUPPORTED EXERCISE AND BMD}

Athletes who participate in sports that involve weight-supported (e.g., non-weight-bearing) exercise, such as cyclists and swimmers, have commonly been found to have low BMD levels. Because these activities generate relatively high levels of muscle forces but no impact forces, this may be viewed as evidence for the importance of gravitational loading. Nikander et al. (31) evaluated femoral neck BMD and parameters of the hip structure in female athletes who participated in impact (i.e., volleyball, hurdling, squash, soccer, speed skating), no/low-impact weight-bearing (i.e., weight lifting, orienteering, skiing), or noimpact weight-supported (i.e., cycling, swimming) sports. As expected, cyclists and swimmers had the lowest BMD levels. However, only the athletes who participated in impact sports had high levels of both femoral neck BMD and section modulus; the latter is an index of the strength of bone against bending. Athletes who participated in low/noimpact weight-bearing activities had increased BMD levels but not section modulus levels. A follow-up study by this research group suggests that impact exercises may specifically generate an increase in cortical thickness (30). Thus, impact activities, through either ground- or joint-reaction forces they generate, seem to confer a unique benefit on bone strength.

It is important to acknowledge that the relatively low BMD levels observed in some athletes, such as cyclists, swimmers, and even long-distance runners (12,29,31), may result from factors other than the loading characteristics of the activity. A prospective study of competitive male road cyclists through $1 \mathrm{yr}$ of training and competition found significant decreases in BMD of the total hip and its subregions and a trend $(P=0.08)$ for a decrease in lumbar spine BMD; average changes in BMD were $-0.7 \%$ to $-1.5 \%$. Although the study did not include a control group, young healthy men would not be expected to have a significant decrease in BMD for $1 \mathrm{yr}$. This raises the possibility that, under certain conditions, exercise may have deleterious, rather than beneficial, effects on the skeleton.

It has been estimated that dermal calcium loss (i.e., sweating) during moderate to vigorous exercise is $\sim 70 \mathrm{mg} \cdot \mathrm{h}^{-1}$ (2). Thus, competitive cyclists and swimmers, who typically train more hours per week than athletes in other sports, may have increased calcium requirements. However, other metabolic factors related to dermal calcium loss during exercise may trigger bone loss. The authors postulate that the dermal loss of calcium during exercise results in a decrease in serum ionized calcium. Because serum calcium level is vigorously defended, such decreases would be expected to trigger an increase in parathyroid hormone (PTH) to mobilize calcium from the skeleton and prevent a further decline in serum calcium. In support of this hypothesis, we (1) and others (11) have observed a significant increase in PTH during exercise that was attenuated by the consumption of calcium-enriched water (11). Thus, it is possible that repeated prolonged bouts of relatively vigorous exercise trigger increases in bone resorption that offset the beneficial mechanical loading effects of exercise. Under this paradigm, the deleterious effects of exercise on bone would be expected to be more prominent if the types of loading forces generated during exercise are not optimal, which may be the case for cycling and swimming.

In addition to dermal calcium loss during exercise, other factors could stimulate excess bone resorption. A detailed discussion of this topic is outside the scope of this article. However, some potential factors include chronic low energy availability, disruptions in gonadal function, nutrient deficiency, and exercise-induced increases in proresorptive cytokines.

\section{PHYSICAL ACTIVITY AND FRACTURE RISK}

There is growing evidence that being physically active reduces the risk for osteoporotic fracture. Several prospective cohort and case-control studies $(5,8-10,14-16,18,27)$ 
categorized participants by level of physical activity and found that the most active subgroup had a reduced risk for hip fracture risk when compared with the least active subgroup (Fig. 3) (32). It seems likely that impact activities play a key role in this fracture prevention, if only because walking is common to many forms of physical activity.

One prospective cohort study in particular, the Nurses' Health Study (9), provided key insights into the importance of impact activities on hip fracture risk. Physical activity was assessed by self-report in more than 60,000 postmenopausal women, aged 40 to $77 \mathrm{yr}$, and incident hip fracture was assessed during $\sim 12$ yr of follow-up. Participants were divided into five groups by physical activity level. Women in the most active group ( $\left.\geq 24 \mathrm{MET} \cdot \mathrm{h} \cdot \mathrm{wk}{ }^{-1}\right)$ had a 55\% lower hip fracture risk than those in the least active group $\left(<3 \mathrm{MET} \cdot \mathrm{h} \cdot \mathrm{wk}^{-1}\right)$. The dose-dependent association between physical activity and hip fracture risk suggested that fracture risk decreased by $6 \%$ for every $3-\mathrm{MET} \cdot \mathrm{h} \cdot \mathrm{wk}^{-1}$ increase in physical activity. Walking was the most commonly reported type of physical activity, and hip fracture risk was $41 \%$ lower in women who reported walking for $4 \mathrm{~h} \cdot \mathrm{wk}^{-1}$ or more when compared with those who reported walking less than $1 \mathrm{~h} \cdot \mathrm{wk}^{-1}$. Walking pace (easy vs average vs brisk to very brisk) was also significantly associated with fracture risk, with the fastest walkers having a 65\% lower risk than the slowest walkers. Importantly, when both walking duration and pace were analyzed in a multivariate model, pace remained a significant predictor of hip fracture risk but duration did not. Because ground-reaction forces increase as walking speed increases, this suggests that impact forces play an important role in the maintenance of bone health. However, even low-impact gravitational loading may have beneficial skeletal effects, because standing for $40 \mathrm{~h} \cdot \mathrm{wk}^{-1}$ or more (which presumably included time spent walking) was associated with a $34 \%$ to $46 \%$ lower risk of hip fracture (9).

A recent meta-analysis concluded that moderate to vigorous physical activity is associated with $45 \%$ reduction in hip fracture risk in women and a 38\% reduction in men (28). Although there have been no randomized controlled studies of the effects of physical activity on fracture prevention, the findings from multiple large observational studies are establishing an evidence base for the antifracture benefits of physical activity. Because commonly performed physical activities (e.g., walking) typically generate both gravitational and muscle forces, the type of loading force underlying the benefit cannot be isolated. To the best of the authors' knowledge, no observational study has investigated whether activities that involve only muscle forces have antifracture efficacy. In fact, in the Nurses' Health Study, which was one of the largest observational studies to examine the association of physical activity with fracture risk, no activity other than walking was reported with sufficient frequency for separate analysis (9).

\section{PHYSICAL ACTIVITY AND BODY COMPOSITION}

If muscle forces are, indeed, the primary mediators of the antifracture benefits that have been observed in physically activity cohorts (Fig. 3), such forces do not seem to be of sufficient magnitude to preserve fat-free mass. Swiss investigators measured body composition by bioelectrical impedance in large groups of women $(n=3184)$ and men $(\mathrm{n}=$ 3549), aged 18 to $98 \mathrm{yr}$, who were categorized as either sedentary or physically active (19). Those in the physically active group reported that they regularly performed moderate to vigorous activities (Q4 MET) at least $3 \mathrm{~h} \cdot \mathrm{wk}^{-1}$ for longer than 2 months. The plots of fatfree mass for women and men grouped by decade of age (Fig. 4) suggest that being physically active has little impact on the age-related decline in fat-free mass. In fact, for both women and men across all age groups, those who were sedentary tended to have higher levels of fat-free mass than those who were physically active, which is likely a result of the sedentary groups needing a larger muscle mass to support their higher levels of adiposity. It is possible that being physically active will not slow the age-related decline in muscle mass 
unless resistance types of activities are performed (7). In the Swiss cohort, resistance activities accounted for less than $10 \%$ of all physical activity and could not be analyzed separately. This low prevalence of resistance exercise is comparable to the estimate that $10 \%$ to $20 \%$ of middle-aged and older women and men in the United States perform resistance exercises at least $2 \mathrm{~d} \cdot \mathrm{wk}^{-1}(6)$.

\section{CONCLUSIONS}

There is no conclusive evidence from clinical studies that either muscle forces or gravitational forces play a more prominent role in regulating bone metabolism. When exercise training programs that included activities that generated ground-impact forces (e.g., walking, running, stairs) were compared with those that did not (e.g., weight-lifting, rowing), both were found to have beneficial effects on BMD $(17,35)$. The increases in BMD in response to impact exercise occurred in the absence of improvements in muscle mass or strength, which argues against muscle forces playing a dominant role. Further evidence that impact forces generate unique skeletal adaptations comes from cross-sectional comparisons of athletes from different sports. Whereas sports that involve either impact forces (e.g., volleyball, orienteering) or no-impact high-magnitude muscle forces (e.g., weight lifting) are both associated with high BMD levels, only sports that involve impact forces seem to have benefits on structural indices of bone strength, possibly by increasing cortical thickness $(30,31)$.

Although the relative effectiveness of gravitational versus muscle loading may not be well understood, there is growing evidence for a dose-response effect of physical activity $(28,32)$, and walking specifically $(9)$, to reduce the risk for hip fracture. The importance of mechanical loading for the prevention of fractures is probably best gleaned from studies of animals, in which small increases in BMC and BMD (5\% to 7\%) in response to mechanical loading translate into very large increases (64\% to $94 \%$ ) in bone strength and resistance to fracture (37). In contrast, pharmacologic therapies that have antifracture in humans generate somewhat larger increases in BMC and BMD (9\% to 15\%) but result in only proportional increases in resistance to fracture ( $7 \%$ to $21 \%)(13,26)$. Further clinical research will be necessary to understand the potential of various types of physical activity to maintain bone health.

\section{Acknowledgments}

Part of the research reported in this article was supported by the National Institutes of Health (R01 AG018198, R01 AG018857, R01 AG019339, P30 DK048520, and M01 RR000051). The results of the present study do not constitute endorsement by the ACSM.

\section{REFERENCES}

1. Barry DW, Kohrt WM. Acute effects of 2 hours of moderate-intensity cycling on serum parathyroid hormone and calcium. Calcif Tissue Int 2007;80:359-365. [PubMed: 17549534]

2. Barry DW, Kohrt WM. BMD decreases over the course of a year in competitive male cyclists. J Bone Miner Res 2008;23:484-491. [PubMed: 18072875]

3. Bergmann G, Graichen F, Rohlmann A. Hip joint loading during walking and running, measured in two patients. J Biomech 1993;26:969-990. [PubMed: 8349721]

4. Binder EF, Kohrt WM. Relationships between body composition and bone mineral content and density in older women and men. Clin Exerc Physiol 2000;2:84-91.

5. Boonyaratavej N, Suriyawongpaisal P, Takkinsatien A, et al. Physical activity and risk factors for hip fractures in Thai women. Osteoporos Int 2001;12:244-248. [PubMed: 11315244]

6. Centers for Disease Control and Prevention. Trends in strength training-United States, 1998-2004. MMWR Morb Mortal Wkly Rep 2006;55:769-772. [PubMed: 16855525] 
7. Evans WJ. Protein nutrition, exercise and aging. J Am Coll Nutr 2004;23:601S-609S. [PubMed: 15640513]

8. Farahmand BY, Persson PG, Michaelsson K, et al. Physical activity and hip fracture: a populationbased case-control study. Int J Epidemiol 2000;29:308-314. [PubMed: 10817130]

9. Feskanich D, Willett W, Colditz G. Walking and leisure-time activity and risk of hip fracture in postmenopausal women. JAMA 2002;288:2300-2306. [PubMed: 12425707]

10. Gregg EW, Cauley JA, Seeley DG, et al. Physical activity and osteoporotic fracture risk in older women. Study of Osteoporotic Fractures Research Group. Ann Intern Med 1998;129:81-88. [PubMed: 9669990]

11. Guillemant J, Le HT, Guillemant S, et al. Acute effects induced by a calcium-rich mineral water on calcium metabolism and on parathyroid function. Osteoporos Int 1997;7:85-86. [PubMed: 9102070]

12. Hind K, Truscott JG, Evans JA. Low lumbar spine bone mineral density in both male and female endurance runners. Bone 2006;39:880-885. [PubMed: 16682267]

13. Hirano T, Burr DB, Turner CH, et al. Anabolic effects of human biosynthetic parathyroid hormone fragment (1-34), LY333334, on remodeling and mechanical properties of cortical bone in rabbits. J Bone Miner Res 1999;14:536-545. [PubMed: 10234574]

14. Hoidrup S, Sorensen TI, Stroger U, et al. Leisure-time physical activity levels and changes in relation to risk of hip fracture in men and women. Am J Epidemiol 2001;154:60-68. [PubMed: 11427405]

15. Jaglal SB, Kreiger N, Darlington GA. Lifetime occupational physical activity and risk of hip fracture in women. Ann Epidemiol 1995;5:321-324. [PubMed: 8520716]

16. Kanis J, Johnell O, Gullberg B, et al. Risk factors for hip fracture in men from southern Europe: the MEDOS study. Osteoporosis Int 1999;9:45-54.

17. Kohrt WM, Ehsani AA, Birge SJ Jr. Effects of exercise involving predominantly either jointreaction or ground-reaction forces on bone mineral density in older women. J Bone Miner Res 1997;12:1253-1261. [PubMed: 9258756]

18. Kujala UM, Kaprio J, Kannus P, et al. Physical activity and osteoporotic hip fracture risk in men. Arch Intern Med 2000;160:705-708. [PubMed: 10724057]

19. Kyle UG, Genton L, Gremion G, et al. Aging, physical activity and height-normalized body composition parameters. Clin Nutr 2004;23:79-88. [PubMed: 14757396]

20. Lang T, LeBlanc A, Evans H, et al. Cortical and trabecular bone mineral loss from the spine and hip in long-duration spaceflight. J Bone Miner Res 2004;19:1006-1012. [PubMed: 15125798]

21. LeBlanc AD, Schneider VS, Evans HJ, et al. Bone mineral loss and recovery after 17 weeks of bed rest. J Bone Miner Res 1990;5:843-850. [PubMed: 2239368]

22. LeBlanc AD, Spector ER, Evans HJ, et al. Skeletal responses to space flight and the bed rest analog: a review. J Musculoskelet Neuronal Interact 2007;7:33-47. [PubMed: 17396004]

23. Martyn-St James M, Carroll S. A meta-analysis of impact exercise on postmenopausal bone loss: the case for mixed loading exercise programmes. Br J Sports Med. in press.

24. Martyn-St James M, Carroll S. High-intensity resistance training and postmenopausal bone loss: a meta-analysis. Osteoporos Int 2006;17:1225-1240. [PubMed: 16823548]

25. Martyn-St James M, Carroll S. Progressive high-intensity resistance training and bone mineral density changes among premenopausal women: evidence of discordant site-specific skeletal effects. Sports Med 2006;36:683-704. [PubMed: 16869710]

26. Mashiba T, Turner CH, Hirano T, et al. Effects of suppressed bone turnover by bisphosphonates on microdamage accumulation and biomechanical properties in clinically relevant skeletal sites in beagles. Bone 2001;28:524-531. [PubMed: 11344052]

27. Michaelsson K, Olofsson H, Jensevik K, et al. Leisure physical activity and the risk of fracture in men. PLoS Med 2007;4:1094-1100.

28. Moayyeri A. The association between physical activity and osteoporotic fractures: a review of the evidence and implications for future research. Ann Epidemiol 2008;18:827-835. [PubMed: 18809340] 
29. Nichols JF, Palmer JE, Levy SS. Low bone mineral density in highly trained male master cyclists. Osteoporos Int 2003;14:644-649. [PubMed: 12856112]

30. Nikander R, Kannus P, Dastidar P, et al. Targeted exercises against hip fragility. Osteoporos Int 2009;20:1321-1328. [PubMed: 19002370]

31. Nikander R, Sievanen H, Heinonen A, et al. Femoral neck structure in adult female athletes subjected to different loading modalities. J Bone Miner Res 2005;20:520-528. [PubMed: 15746998]

32. Physical Activity Guidelines Advisory Committee Report. Washington (DC): US Department of Health and Human Services; 2008. Physical Activity Guidelines Advisory Committee.

33. Shackelford LC, LeBlanc AD, Driscoll TB, et al. Resistance exercise as a countermeasure to disuse-induced bone loss. J Appl Physiol 2004;97:119-129. [PubMed: 15220316]

34. Smith SM, Zwart SR, Heer M, et al. WISE-2005: supine treadmill exercise within lower body negative pressure and flywheel resistive exercise as a countermeasure to bed rest-induced bone loss in women during 60-day simulated microgravity. Bone 2008;42:572-581. [PubMed: 18249055]

35. Snow-Harter C, Bouxsein ML, Lewis BT, et al. Effects of resistance and endurance exercise on bone mineral status of young women: a randomized exercise intervention trial. J Bone Miner Res 1992;7:761-769. [PubMed: 1642145]

36. Trappe TA, Burd NA, Louis ES, et al. Influence of concurrent exercise or nutrition countermeasures on thigh and calf muscle size and function during 60 days of bed rest in women. Acta Physiol (Oxf) 2007;191:147-159. [PubMed: 17655736]

37. Turner $\mathrm{CH}$, Robling AG. Designing exercise regimens to increase bone strength. Exerc Sport Sci Rev 2003;31:45-50. [PubMed: 12562170] 


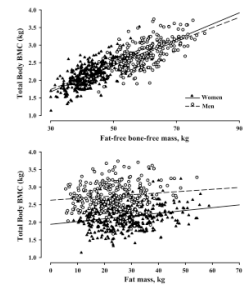

FIGURE 1.

Associations of TBBMC with fat-free mass (top panel) and fat mass (bottom panel) in 685 women (triangles) and men (circles), aged 21 to 90 yr. Regression lines were generated separately for women (solid lines) and men (broken lines). 


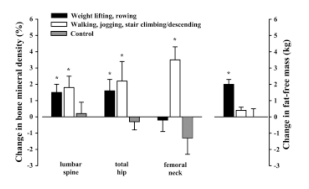

FIGURE 2.

Changes in bone mineral density (\%; left panel) and fat-free mass ( $\mathrm{kg}$; right panel) of postmenopausal women in response to 9 months of low-impact (i.e., weight lifting, rowing) or high-impact (i.e., walking, jogging, stair climbing/descending) exercise training versus a no-exercise control group. *Different from control, $P<0.05$. [Adapted from Kohrt WM, Ehsani AA, Birge SJ Jr. Effects of exercise involving predominantly either joint-reaction or ground-reaction forces on bone mineral density in older women. J Bone Miner Res. 1997;12:1253-61. Copyright (C) 1997 American Society for Bone and Mineral Research. Used with permission.] 


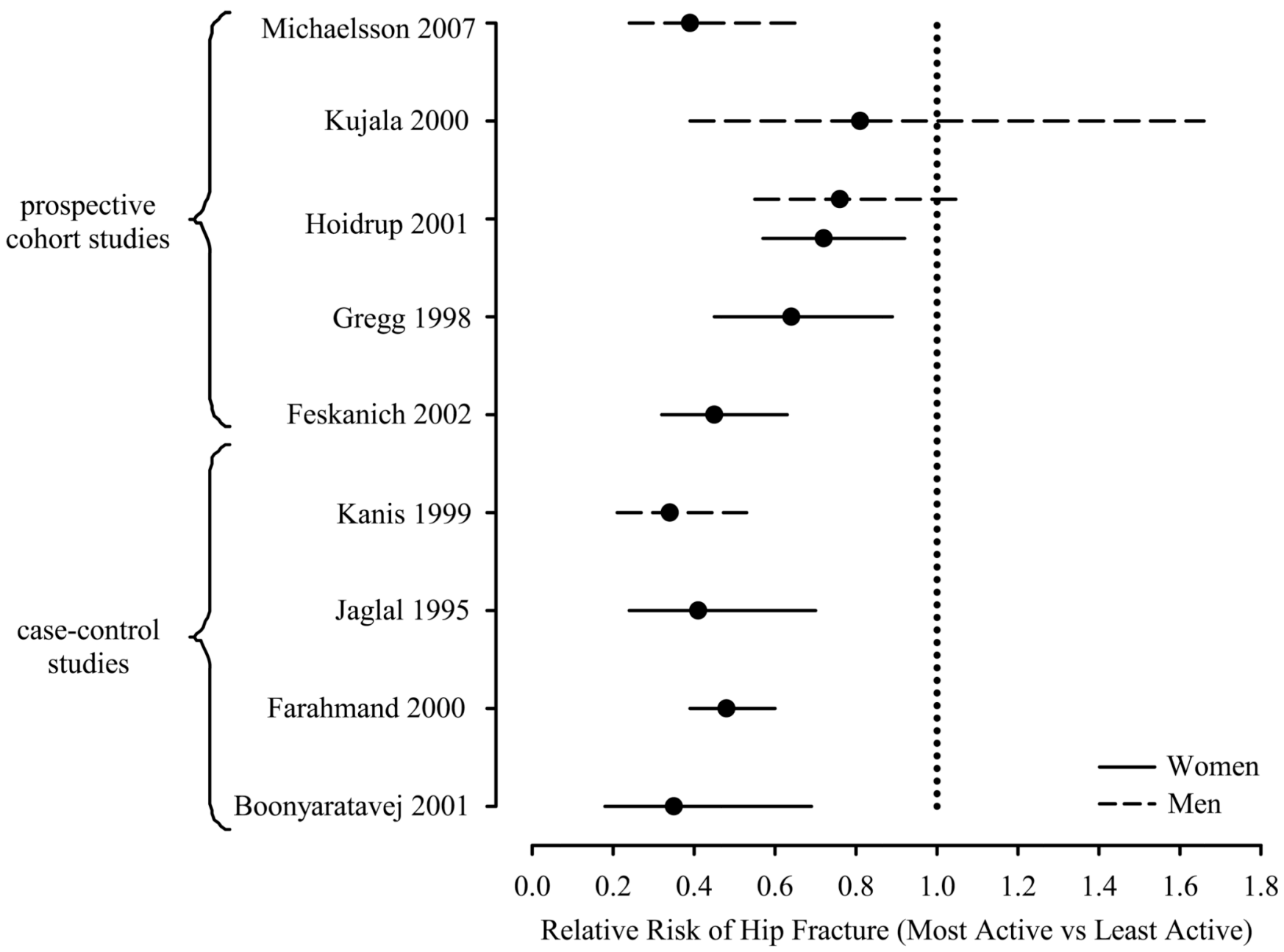

FIGURE 3.

Point estimates of relative risk ( $\pm 95 \%$ confidence intervals) of hip fracture from observational studies that examined multiple levels of physical activity (most active group vs least active group). [Adapted from Physical Activity Guidelines Advisory Committee. Physical Activity Guidelines Advisory Committee Report. Washington (DC): US Department of Health and Human Services (32).] 

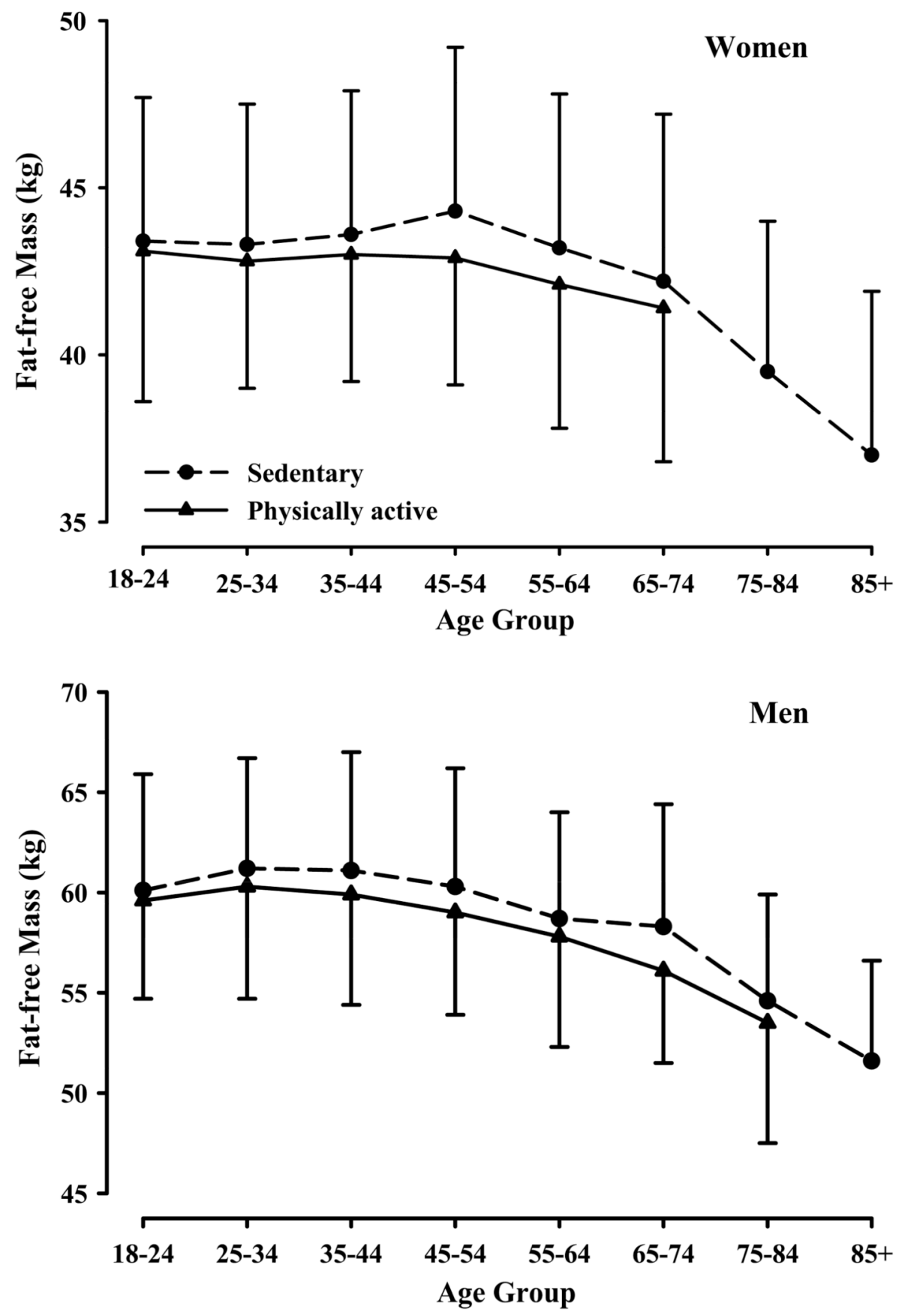

FIGURE 4.

Age-related changes in fat-free mass (mean \pm SD) in sedentary and physically active women (top panel) and men (bottom panel). [Adapted from Kyle UG, Genton L, Gremion G, et al. Aging, physical activity and height-normalized body composition parameters. Clin Nutr. 2004;23:79-88.] 